\title{
Idiopathic pleuroparenchymal fibroelastosis: consideration of a clinicopathological entity in a series of Japanese patients
}

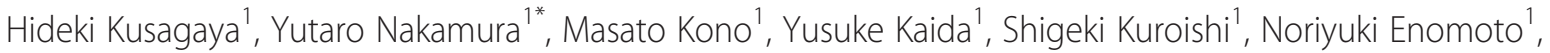 \\ Tomoyuki Fujisawa ${ }^{1,2}$, Naoki Koshimizu' ${ }^{1}$, Koshi Yokomura', Naoki Inui ${ }^{1,3}$, Takafumi Suda ${ }^{1}$, Thomas V Colby ${ }^{4}$ \\ and Kingo Chida ${ }^{1}$
}

\begin{abstract}
Background: Idiopathic pleuroparenchymal fibroelastosis (IPPFE) is a recently reported group of disorders characterized by fibrotic thickening of the pleural and subpleural parenchyma predominantly in the upper lobes. We report five Japanese cases fulfilling the criteria of IPPFE and address whether it should be considered a separate clinicopathologic entity. And this study was an attempt to identify features in common between IPPFE and previously described idiopathic upper lobe fibrosis (IPUF), allowing IPPFE to be considered as a distinct entity in our Japanese series.

Methods: Five consecutive cases of idiopathic interstitial lung disease confirmed as IPPFE by surgical lung biopsy were studied.

Results: There were four males and one female, aged $70 \pm 2.76 \mathrm{yr}$. No associated disorder or presumed cause was found in any case. Lung function tests found a restrictive ventilatory defect (4/5) and/or impairment of DLco (4/5). Chest X-ray showed marked apical pleural thickening in all cases. Computed tomography of the chest in all cases mainly showed intense pleural thickening and volume loss associated with evidence of fibrosis, predominantly in the upper lobes. In all cases in this study, markedly thickened visceral pleura and prominent subpleural fibrosis characterized by both elastic tissue and dense collagen were clearly shown. All cases were alive at the last follow-up, 17.6 \pm 13.59 months after diagnosis; however, all had deteriorated both clinically and radiologically.
\end{abstract}

Conclusions: IPPFE deserves to be defined as a separate, original clinicopathologic entity owing to its uniformity and IPPFE has some features in common with previously described idiopathic upper lobe fibrosis (IPUF). Our limited experience with a cohort of 5 subjects suggests that IPPFE can be rapidly progressive.

Keywords: Idiopathic interstitial lung disease, Pleural fibrosis, Fibroelastosis, Pleuroparenchymal fibroelastosis

\section{Background}

Idiopathic pleuroparenchymal fibroelastosis (IPPFE) is a recently reported rare disorder characterized by fibrotic thickening of the pleural and subpleural parenchyma predominantly in the upper lobes [1]; as such IPPFE is distinct from other types of idiopathic interstitial pneumonia [2]. Since 1992 several reports of upper lobepredominant pulmonary fibrosis (Idiopathic upper lobe

\footnotetext{
* Correspondence: nakayuta@hama-med.ac.jp

${ }^{1}$ Second Division, Department of Internal Medicine, Hamamatsu University School of Medicine, Hamamatsu, Japan

Full list of author information is available at the end of the article
}

fibrosis/IPUF) have been reported in the Japanese literature [3-9] and some of these cases share features with IPPFE. Herein we report five Japanese cases of IPPFE, and describe the clinical, radiologic and evolutionary profile of IPPFE in a retrospective series in order to determine if IPPFE represents a separate clinicopathologic entity and to clarify its relationship with IPUF.

\section{Methods}

The subjects included 5 patients fulfilling criteria IPPFE diagnosed by surgical lung biopsy [1] (SLB) from May 2009 to Sep 2011 at Hamamatsu University Hospital and 
affiliated hospitals in Japan. Lung biopsies were performed by video-assisted thoracoscopy, and specimens were obtained from 2 or more lobes in every case. We used the pathological criteria for the diagnosis of IPPFE as follows: (1) intense fibrosis of the visceral pleura; (2) prominent, homogenous, subpleural fibroelastosis; (3) sparing of the parenchyma distant from the pleura; (4) mild, patchy lymphoplasmocytic infiltrates; and (5) small numbers of fibroblastic foci present. All of our reported cases met all of the criteria. This criteria is based on the paper by Frankel et al. [1]. Surgical lung specimens were also reviewed by an expert pulmonary pathologist (T.V.C.). This study was approved by the Institutional Review Board of Hamamatsu University School of Medicine. Clinical data were obtained from case medical records. Laboratory findings and pulmonary function test results at the time of SLB were also recorded.

\section{Results}

\section{Clinical features and biology}

Table 1 shows the clinical characteristics and laboratory data. All patients were more than 60 years of age. There were no current smokers, and only one patient was an ex-smoker. Four out of five were classified as underweight by body mass index (BMI). The main presenting symptom was dyspnea. There was no significant family or occupational history. Case 1 had a history of gingival cancer, and had chemotherapy. Mild lymphocytosis was found on BAL in one case. Examination and cultures of lung tissue for bacteria, mycobacteria, fungi, parasites, and viruses were negative in all cases. KL- 6 was elevated in two cases and normal in three. Interestingly, the level of SP-D was higher than the normal range in all the cases.

\section{Pulmonary function test}

All cases underwent a spirometry test and measurement of total lung capacity (TLC). A restrictive ventilatory defect defined by $\% \mathrm{FVC}<80 \%$ was found in 4 cases. In addition, four cases had impairment of DLco (Table 2).

\section{Radiological features}

Chest radiographs in all cases showed marked apical pleural thickening and superior hilar retraction (Figure 1). Right lung is predominantly affected in case 2, 3, and 5, left lung in case 4. High-resolution CT (HRCT) showed intense pleural thickening associated with evidence of fibrosis. In Case 1 and 2, upper lobe volume loss, architectural distortion and traction bronchiectasis were also prominent.

\section{Pathological features}

In all cases in this study, the histopathological findings fitted those previously described for IPPFE. Markedly thickened visceral pleura and prominent subpleural fibrosis characterized by both elastic tissue and dense collagen were clearly shown (Figures $2 \mathrm{a}, \mathrm{b}$ and $3 \mathrm{a}, \mathrm{b}$ ). The border between the fibroelastosis and the underlying normal lung parenchyma was abrupt, and the parenchyma distant from the pleura was spared (Figure 2a). Fibroblastic foci were rarely noted at the leading edge of the fibrosis. Inflammation was variable and primarily consisted of small aggregates of lymphocytes. Asbestos bodies were absent.

\section{Treatment and clinical outcome}

All cases initially had no treatment. The mean duration of follow-up after the pathologic diagnosis was 12.1 months (range 4.4 - 22). The total duration of follow-up from the onset of the symptoms to the last

Table 1 Clinical characteristics and laboratory data

\begin{tabular}{|c|c|c|c|c|c|}
\hline & Case. 1 & Case. 2 & Case. 3 & Case. 4 & Case.5 \\
\hline Age (yr)/sex & 70, male & 74, female & 67, male & 67, male & 72, male \\
\hline Smoking status & Ex-smoker & Never-smoker & Never-smoker & Never-smoker & Never-smoker \\
\hline $\mathrm{BMI}$ & 18.7 & 17.5 & 15.9 & 17.9 & 17.0 \\
\hline Symptom & Dyspnea & Dyspnea & Cough & Dyspnea & Dyspnea \\
\hline Crackles & + & - & - & - & + \\
\hline BAL Iym (\%) & 8.6 & 0 & 16 & 1 & ND \\
\hline neu (\%) & 0.6 & 0 & 1.0 & 8 & ND \\
\hline eos (\%) & 0 & 1 & 0 & 3 & ND \\
\hline $\mathrm{KL}-6(\mathrm{U} / \mathrm{ml})$ & 410 & 604 & 437 & 1000 & 469 \\
\hline SP-D (ng/ml) & 204 & 131 & 129 & 437 & 133 \\
\hline $\mathrm{RF}(\mathrm{U} / \mathrm{ml})$ & 54 & - & 4 & 1 & 16 \\
\hline ANA & - & $\times 40$ & $\times 40$ & - & - \\
\hline $\mathrm{PaO}_{2}$ (Torr) & 82 & 94.4 & 95.8 & 83.0 & 82.0 \\
\hline Prognosis & Survive 12 months & Survive 6 months & Survive 44 months & Survive 10 months & Survive 16 months \\
\hline
\end{tabular}

Abbreviations: BMI, body mass index, BAL, broncho-alveolar lavage, KL-6, Sialylated carbohydrate antigen KL-6, Sp-D, Surfactant Protein D, RF, rheumatoid factor, ANA, antinuclear antibody. 
Table 2 Pulmonary function test data

\begin{tabular}{lccccc}
\hline & Case.1 & Case.2 & Case.3 & Case.4 & Case.5 \\
\hline TLC (L), \%predicted (\%) & $4.09(79.4)$ & $2.90(79.0)$ & $5.97(107.4)$ & $3.69(72.1)$ & $2.9(56.9)$ \\
\hline FVC (L), \%predicted (\%) & $2.50(74.1)$ & $1.41(74.1)$ & $3.24(93.6)$ & $2.14(63.7)$ & $2.12(64.0)$ \\
\hline FEV1 (L), \%predicted (\%) & $2.06(77.5)$ & $1.33(87.5)$ & $3.20(101.6)$ & $2.11(79.0)$ & $1.67(64.3)$ \\
\hline FEV1.0/FVC & 90.4 & 95 & 96.7 & 96.3 & 96.5 \\
\hline FRC (L), \%predicted (\%) & $3.0(75.2)$ & $2.1(101)$ & $3.87(85.6)$ & $2.61(67.3)$ & $2.14(51.8)$ \\
\hline RV (L), \%predicted (\%) & $1.97(121.6)$ & $1.42(87.1)$ & $2.82(154.1)$ & $1.55(100)$ & $1.38(84.1)$ \\
\hline RV/TLC (\%), \%predicted (\%) & $48.17(120.2)$ & $48.97(145.1)$ & $47.2(120.7)$ & $42.0(107.2)$ & $47.59(111.2)$ \\
\hline DLCO (mL/min/mmHg), \%predicted (\%) & $9.41(74.2)$ & $9.94(76.8)$ & $10.55(71.3)$ & $12.51(96.1)$ & $6.12(55.1)$ \\
\hline DLCO/NA (mL/min/mmHg), \%predicted (\%) & $3.04(69.1)$ & $4.42(103.3)$ & $2.10(47.1)$ & $4.42(98.4)$ & $2.78(64.5)$
\end{tabular}

Abbreviations: TLC, Total lung capacity, FVC, Forced vital capacity, FEV, Forced expiratory volume, FRC, Functional residual capacity, RV Residual volume, DLco, Diffusing capacity for carbon monoxide, DLco/VA, Diffusing capacity divided by the alveolar volume.

follow-up was 45.2 months (range 7-83). All patients were alive at the last follow-up (Table 2), but all had disease progression according to clinical and lung function tests, especially in terms of \%FVC (Figure 4).

\section{Discussion}

It is clear that IPPFE is not a new pulmonary disease. As Reddy et al [10] reported, it has previously received other names, such as idiopathic upper lobe fibrosis
(IPUF), and has more frequently been described in the Japanese literature [3-5,9] and may be more common in the Japanese. IPPFE has been defined as a distinct entity in the English literature by Frankel et al.; a unique clinical presentation and precise characteristic pathologic criteria are described [1]. Our study was an attempt to identify features in common between these two entities, allowing IPPFE to be considered distinct as it applies to our Japanese series.

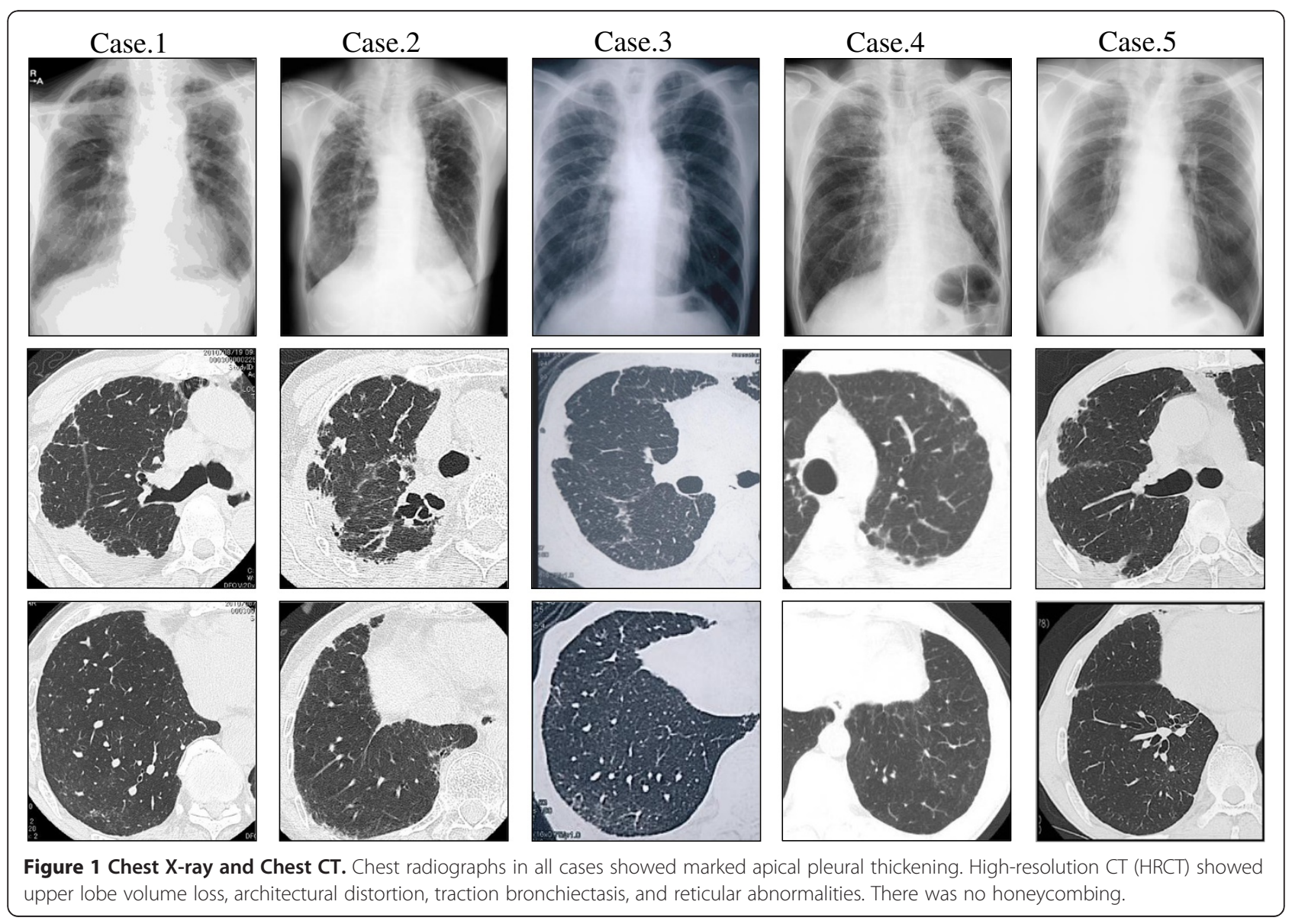



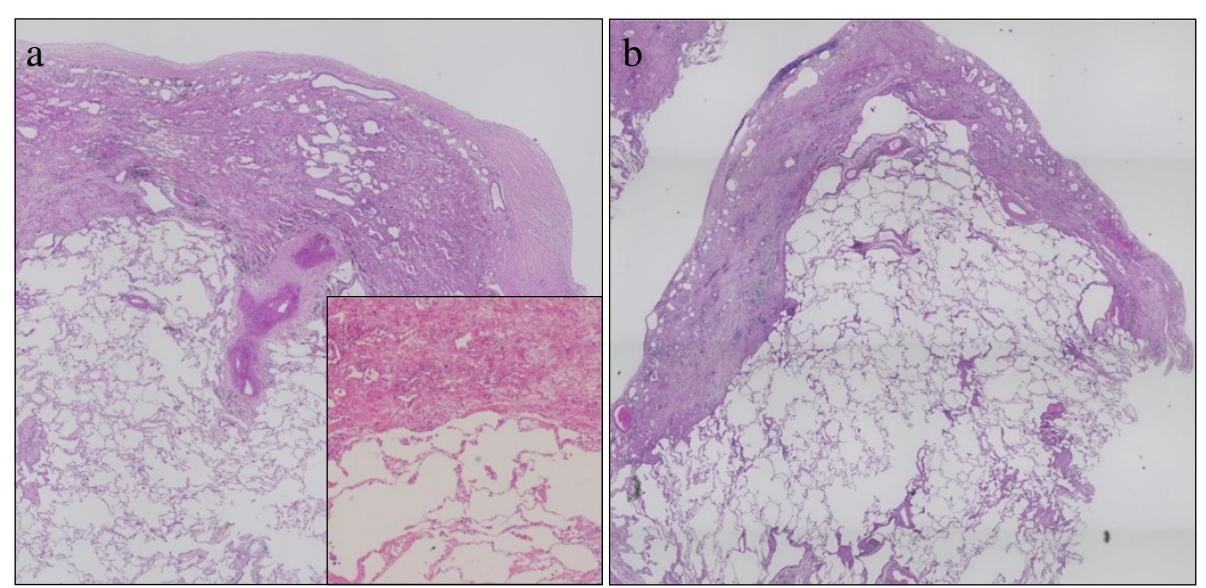

Figure 2 Histopathological Findings (hematoxylin-eosin). Surgical lung biopsy specimen at low power (a:case 1, b:case 5) and high power (a, inset) showed markedly thickened visceral pleura and prominent subpleural fibrosis characterized by abnormal increase of elastic tissue and dense collagen. Abrupt transition to normal parenchyma was also seen. Parenchyma distant from the pleura was spared.

The clinical presentation and the findings of the lung function tests were characteristic of restrictive interstitial lung disease. Radiological features were also characteristic, including intense pleural thickening associated with evidence of fibrosis, most striking pattern in the upper lobes with volume loss and architectural distortion. The upper lobes were always more severely involved, with involvement of the lower lobes being absent or less marked. Piciucchi and colleagues reported that one case had honeycombing on HRCT findings in their cases [11]. In our series, no patients had honeycombing. In all the reports describing PPFE, eight out of total 25 cases (32\%) had a history of malignancy. Among those cases, four cases had treatment with BMT [12] and those cases presented with histological evidence of obliterative bronchiolitis, which was not a feature in those with idiopathic presentation of PPFE, suggesting that bone marrow transplantation is a potential etiology at least in a subgroup of cases with pleuroparenchymal fibroelastosis. In our series, Case 1 had a history of gingival cancer treated with surgery followed by adjuvant chemotherapy. However, no cases had BMT, and other cases never had malignancy.

In terms of therapy and prognosis, previous cases of IPPFE showed a $40 \%$ mortality rate. In addition, many cases gradually deteriorated. In our series, consistent with previous reports, although no death occurred, all the cases clinically and functionally deteriorated even in a relatively short follow-up period. There are no therapeutic options available for IPPFE except for supportive care and ultimately lung transplantation. Regarding the mechanism of elastosis, it has not been determined whether fibroelastosis occurred by overproduction of elastin or impairment of degradation. Pirfenidone is an antifibrotic drug,

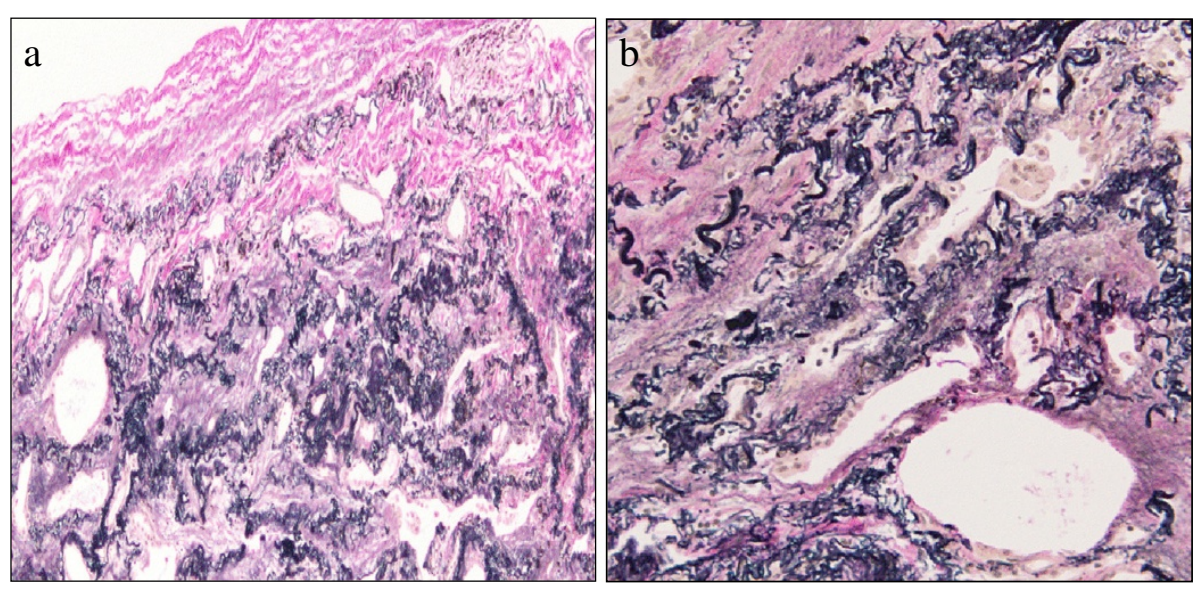

Figure 3 Histopathological Findings (Elastic Van Gieson). Surgical lung biopsy specimen at low power (a) and high power (b) demonstrated an abundance of short, amorphous elastic fibers (Case 1). 

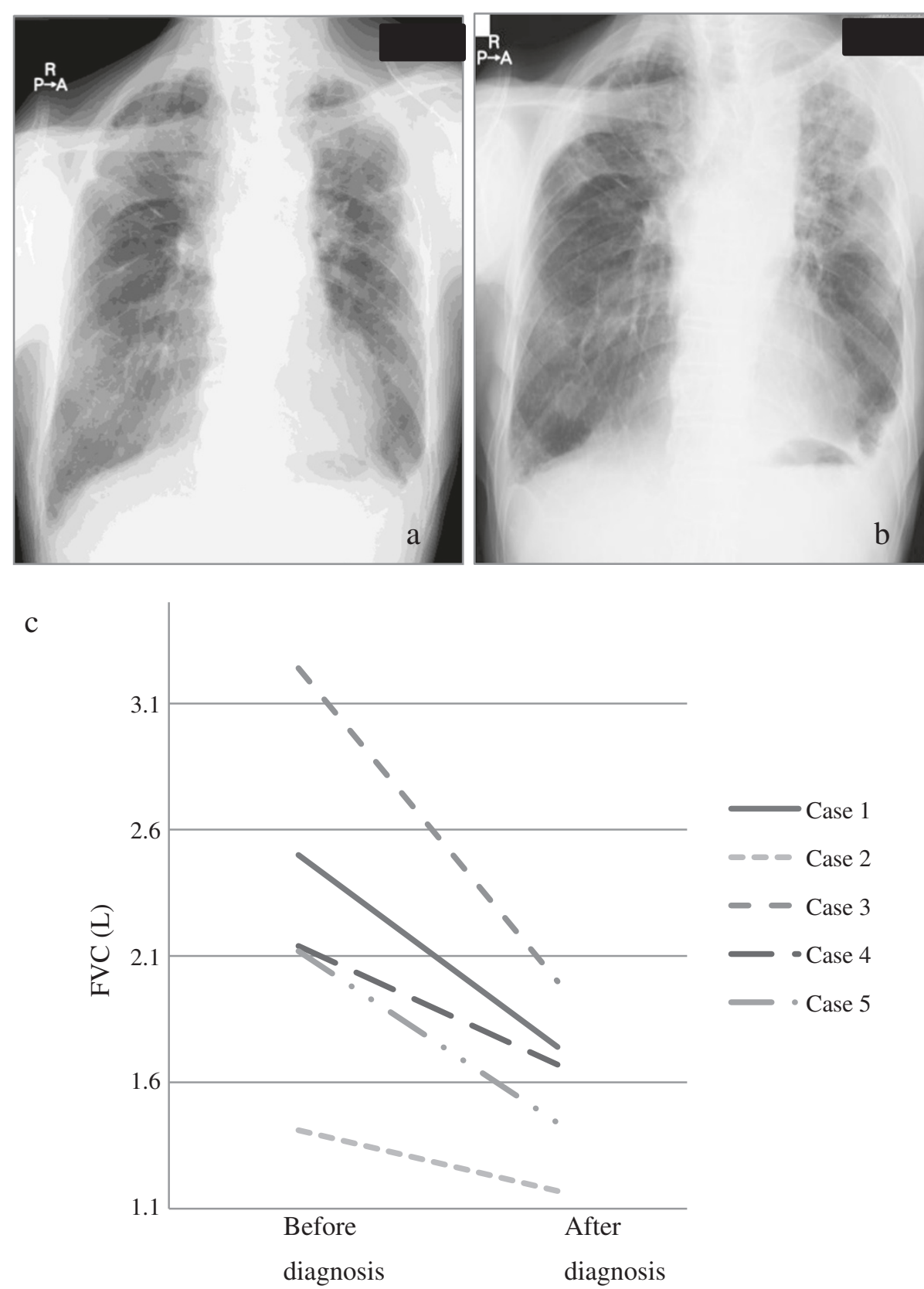

Figure 4 Clinical Course of the cases. Case 1 radiologically deteriorated a year after diagnosis $(\mathbf{a}, \mathbf{b})$. Other cases present similar behavior. (c) Decrease in FVC was confirmed in all cases during the follow up. The median follow up was 12.1 (range 4.37-22.2) months.

currently used only for idiopathic pulmonary fibrosis (IPF) [13-15], which inhibits fibrotic factors, most notably TGF $\beta$. As a consequence, downstream synthesis of extracellular matrix proteins, such as fibronectin, elastin and collagen, is reduced $[16,17]$. Hence, it may potentially be beneficial for cases of IPPFE.

There are several case reports of idiopathic, upper lobepredominant fibrosis in the Japanese language literature [3-6,9]. Among those reports, it has been described that the thinness of the body was a characteristic of the disease.
Consistent with these reports, our cases showed low BMI. However, there are no data on BMI or body weight of patients with IPPFE in reports from outside of Japan. Radiologically upper lobe predominance is associated with both IPUF and IPPFE. As both IPUF and IPPFE are relatively rare and only recently described, there are no guidelines or criteria as to how extensive the opacities in the middle and lower lobes can be. In terms of other aspects of the disease, such as possible occurrence of pneumothorax, infection and slow progression of the disease, 
IPPFE and IPUF seem to be within the same spectrum of disease. Although IPUF patients with surgical lung biopsy or autopsy were reported in some Japanese literature, there are no pathological criterias as IPUF. But in all those cases, intraalveolar fibrosis and subpleural elastosis were noted. Those findings are same to the pathological findings with IPPFE. Interestingly, the specimens from some patients shows UIP like pattern in the lower lobe. Amitani et al. [9] strictly defined the lesion is in the upper lobe, but the report from Shiota et al. [6] include the patients with UIP like lesion. Reddy [10] also mentioned patients with PPFE had the fibrosis in the lower lobe. Further studies are needed what is the possible lesion in mid-lower lobe. Finally, pulmonary surfactant protein D (SP-D) was elevated in all cases in our series. It is expressed in alveolar type II and bronchiolar epithelial cells and is secreted into alveoli and conducting airways $[18,19]$. Our data suggest that those epithelial cells may play a role in the fibrotic process of the diseases.

There are some limitations to the present study. First, there were selection and recall biases because this was a retrospective study. Since the current authors' institutions are regional referral centers for diffuse lung disease, the proportion and severity of the disease reported here may differ substantially from those found in the community. Second, the sample size was too small to determine the precise prevalence and clinical characteristics of IPPFE. Prospective studies incorporating larger series are necessary to verify these results.

\section{Conclusions}

This study shows that the clinical, radiological and pathological presentation of IPPFE is remarkably uniform in cases with apparently idiopathic lung disease. We thus consider that idiopathic PPFE deserves to be defined as a distinct clinicopathological syndrome largely overlapping with previously described IPUF. It should be distinguished from other types of interstitial lung disease, especially because it is highly progressive.

\section{Consent}

Written informed consent was obtained from the patient for publication of this case report and accompanying images. A copy of the written consent is available for review by the Editor-in-Chief of this journal.

\footnotetext{
Abbreviations

BMI: body mass index; BAL: broncho-alveolar lavage; KL-6: sialylated carbohydrate antigen KL-6; Sp-D: surfactant protein D; RF: rheumatoid factor; ANA: antinuclear antibody; TLC: total lung capacity; FVC: forced vital capacity; FEV: forced expiratory volume; FRC: functional residual capacity; RV: residual volume; DLco: diffusing capacity for carbon monoxide; DLco/NA: diffusing capacity divided by the alveolar volume.
}

\section{Competing interests}

All authors declare that we have no competing interests.

\section{Authors' contributions}

HK: Conception and design, Data collection, Data analysis and interpretation, Manuscript writing. YN: Conception and design, Data analysis and interpretation, Manuscript writing, Final approve of manuscript. MK: Data collection. YK: Data collection. TF: Data collection. NE: Data collection. HS: Data collection. NK: Data collection. KY: Data collection. TS: Data analysis. T.V. C: Conception and design, Data analysis and interpretation, Manuscript writing. KC: Conception and design, Administrative support, Data analysis and interpretation. All authors read and approved the final manuscript.

\section{Acknowledgements}

We thank Dr. Takeshi Johkoh (Kinki Central Hospital of Mutual Aid Association of Public School Teachers, Itami City, Japan) for helpful comments. This study was partly supported by a grant to the Diffuse Lung Diseases Research Group from the Ministry of Health, Labour and Welfare, Japan.

\section{Author details}

${ }^{1}$ Second Division, Department of Internal Medicine, Hamamatsu University School of Medicine, Hamamatsu, Japan. ${ }^{2}$ Department of Laboratory Medicine, Hamamatsu University School of Medicine, Hamamatsu, Japan. ${ }^{3}$ Department of Clinical Pharmacology and Therapeutics, Hamamatsu University School of Medicine, Hamamatsu, Japan. ${ }^{4}$ Department of Laboratory Medicine/Pathology, Mayo Clinic Arizona, Scottsdale, Arizona, USA.

Received: 30 May 2012 Accepted: 29 November 2012

Published: 5 December 2012

\section{References}

1. Frankel SK, Cool CD, Lynch DA, et al: Idiopathic pleuroparenchymal fibroelastosis: description of a novel clinicopathologic entity. Chest 2004, 126:2007-2013.

2. American Thoracic Society/European Respiratory Society International Multidisciplinary Consensus Classification of the Idiopathic Interstitial Pneumonias: This joint statement of the American Thoracic Society (ATS), and the European Respiratory Society (ERS) was adopted by the ATS board of directors, June 2001 and by the ERS Executive Committee, June 2001. Am J Respir Crit Care Med 2002, 165:277-304.

3. Nei T, Kawamoto M, Satoh E, et al: A case of suspected idiopathic pulmonary upper lobe fibrosis (Amitani disease) with acute exacerbation. Nihon Kokyuki Gakkai Zasshi 2009, 47:116-121.

4. Morimoto A, Mochizuki Y, Nakahara Y, et al: Case of idiopathic pulmonary upper lobe fibrosis. Nihon Kokyuki Gakkai Zasshi 2010, 48:944-949.

5. Kobayashi Y, Sakurai M, Kushiya M, et al: Idiopathic pulmonary fibrosis of the upper lobe: a case report. Nihon Kokyuki Gakkai Zasshi 1999, 37:812-816.

6. Shiota S, Shimizu K, Suzuki M, et al: Seven cases of marked pulmonary fibrosis in the upper lobe. Nihon Kokyuki Gakkai Zasshi 1999, 37:87-96.

7. A case of idiopathic cavitation of lung demonstrated at the Postgraduate Medical School of London. Br Med J 1966, 1:345-348.

8. Undiagnosable Lung Disease. Br Med J 1962, 1:1403-1410.

9. Amitani RNA, Kuse F: Idiopathic pulmonary upper lobe fibrosis (IPFU). Kokyu 1992, 11:693-699.

10. Reddy TL, Tominaga M, Hansell DM, et al: Pleuroparenchymal fibroelastosis: a spectrum of histopathological and imaging phenotypes. Eur Respir J 2012, in press.

11. Piciucchi S, Tomassetti S, Casoni G, et al: High resolution CT and histological findings in idiopathic pleuroparenchymal fibroelastosis: features and differential diagnosis. Respir Res 2011, 12:111.

12. von der Thusen JH, Hansell DM, Tominaga $M$, et al: Pleuroparenchymal fibroelastosis in patients with pulmonary disease secondary to bone marrow transplantation. Mod Pathol 2011, 24:1633-1639.

13. Azuma A, Nukiwa T, Tsuboi E, et al: Double-blind, placebo-controlled trial of pirfenidone in patients with idiopathic pulmonary fibrosis. Am J Respir Crit Care Med 2005, 171:1040-1047.

14. Taniguchi $H$, Ebina $M$, Kondoh $Y$, et al: Pirfenidone in idiopathic pulmonary fibrosis. Eur Respir J 2010, 35:821-829.

15. Noble PW, Albera C, Bradford WZ, et al: Pirfenidone in patients with idiopathic pulmonary fibrosis (CAPACITY): two randomised trials. Lancet 2011, 377:1760-1769. 
16. Dosanjh A: Pirfenidone: anti-fibrotic agent with a potential therapeutic role in the management of transplantation patients. Eur J Pharmacol 2006, 536:219-222.

17. Dosanjh A, lkonen T, Wan B, et al: Pirfenidone: A novel anti-fibrotic agent and progressive chronic allograft rejection. Pulm Pharmacol Ther 2002, 15:433-437.

18. Greene KE, Wright JR, Steinberg KP, et al: Serial changes in surfactant-associated proteins in lung and serum before and after onset of ARDS. Am J Respir Crit Care Med 1999, 160:1843-1850.

19. Greene KE, King TE Jr, Kuroki Y, et al: Serum surfactant proteins-A and -D as biomarkers in idiopathic pulmonary fibrosis. Eur Respir J 2002, 19:439-446.

doi:10.1186/1471-2466-12-72

Cite this article as: Kusagaya et al:: Idiopathic pleuroparenchymal

fibroelastosis: consideration of a clinicopathological entity in a series of Japanese patients. BMC Pulmonary Medicine 2012 12:72.

\section{Submit your next manuscript to BioMed Central and take full advantage of:}

- Convenient online submission

- Thorough peer review

- No space constraints or color figure charges

- Immediate publication on acceptance

- Inclusion in PubMed, CAS, Scopus and Google Scholar

- Research which is freely available for redistribution 